\title{
Interactive 3D Visual Analysis of Atmospheric Fronts
}

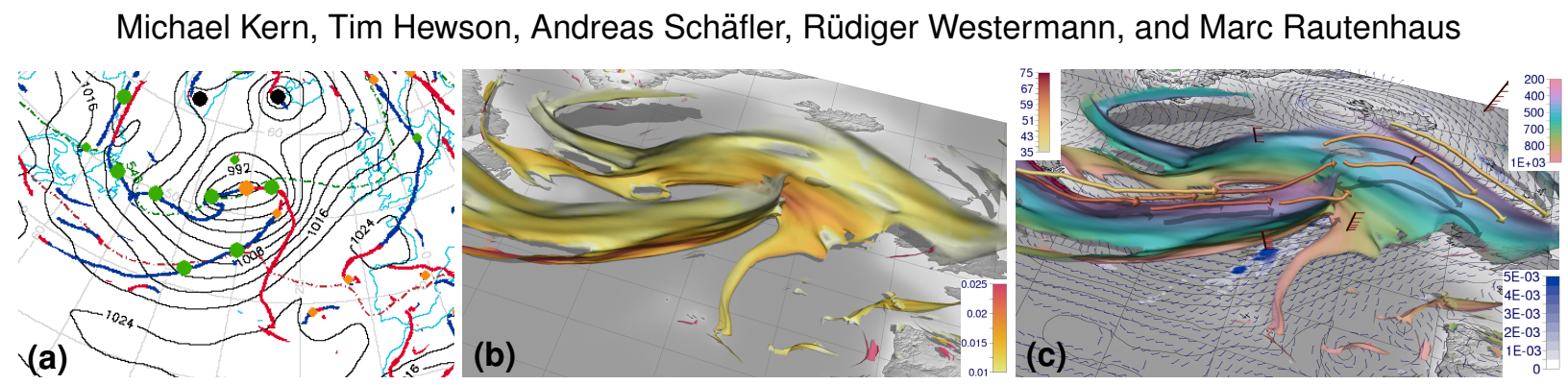

Fig. 1. Cyclone "Vladiana", 00:00 UTC 23 September 2016. (a) Objectively identified fronts at $1 \mathrm{~km}$ above ground from ECMWF operations, using the algorithm described in [15]. (b) 3D fronts identified and visualized with our method, color denotes frontal strength $\left(\mathrm{K} \mathrm{km}^{-1}\right)$. (c) 3D fronts combined with further meteorological fields and features. Front color denotes pressure (hPa). Overlain are jet-stream core lines detected and visualized with the approach by Kern et al. [19], colored by wind speed $\left(\mathrm{m} \mathrm{s}^{-1}\right)$. Surface contours show mean sea level pressure. Blue surface color shows precipitation $\left(\mathrm{m} \mathrm{h}^{-1}\right)$. Wind barbs show surface wind.

\begin{abstract}
Atmospheric fronts play a central role in meteorology, as the boundaries between different air masses and as fundamental features of extra-tropical cyclones. They appear in numerous conceptual model depictions of extra-tropical weather systems. Conceptually, fronts are three-dimensional surfaces in space possessing an innate structural complexity, yet in meteorology, both manual and objective identification and depiction have historically focused on the structure in two dimensions. In this work, we -a team of visualization scientists and meteorologists- propose a novel visualization approach to analyze the three-dimensional structure of atmospheric fronts and related physical and dynamical processes. We build upon existing approaches to objectively identify fronts as lines in two dimensions and extend these to obtain frontal surfaces in three dimensions, using the magnitude of temperature change along the gradient of a moist potential temperature field as the primary identifying factor. We introduce the use of normal curves in the temperature gradient field to visualize a frontal zone (i.e., the transitional zone between the air masses) and the distribution of atmospheric variables in such zones. To enable for the first time a statistical analysis of frontal zones, we present a new approach to obtain the volume enclosed by a zone, by classifying grid boxes that intersect with normal curves emanating from a selected front. We introduce our method by means of an idealized numerical simulation and demonstrate its use with two real-world cases using numerical weather prediction data.
\end{abstract}

Index Terms-Meteorology, Atmospheric Fronts, Feature Detection

\section{INTRODUCTION}

In meteorology, fronts separate atmospheric air masses of different characteristics (e.g., warm and humid versus cold and dry; see, e.g., [56]). Indeed, fronts are among the most important features used in weather forecasting due to the associated weather activity, ranging from temperature changes to severe weather. They are most commonly denoted by manually-analyzed 2D line segments on weather maps. Ordinarily such lines represent fronts at the surface; an automatically generated example is provided in Fig. 1a. Conceptually, however, fronts are surfaces in $3 \mathrm{D}$ space, yet only very occasionally can one see fronts at upper levels marked on a standard chart. This is in spite of regular references to the importance of the vertical structure for surface weather $[2,6,22,24]$. Identifying fronts and judging their 3D temporal evolution can thus be crucial for weather forecasting; however,

- Michael Kern, Rüdiger Westermann and Marc Rautenhaus" are with the Computer Graphics \& Visualization Group, Technische Universität München, Garching, Germany. ("M.R. is now with Universität Hamburg, Regional Computing Center, Hamburg, Germany.)

E-mail: \{michi.kern,westermann, marc.rautenhaus\}@tum.de

- Tim Hewson is with the European Centre for Medium-Range Weather Forecasts, Reading, UK. E-mail: tim.hewson@ecmwf.int

- Andreas Schäfler is with the Deutsches Zentrum für Luft- und Raumfahrt (DLR), Oberpfaffenhofen, Germany. E-mail: andreas.schaefler@dlr.de

Manuscript received xx xxx. 201x; accepted xx xxx. 201x. Date of Publication xx xxx. 201x; date of current version xx xxx. 201x. For information on obtaining reprints of this article, please send e-mail to: reprints@ieee.org. Digital Object Identifier: $x x . x x x x / T V C G .201 x . x x x x x x x$ analysts currently lack the tools and the time to investigate this in detail. Frontal structures have also been extensively studied in atmospheric research, mainly using manual analysis of 2D sections of related fields (including temperature, humidity, wind; e.g., [11, 12, 22, 23, 29, 48]), with a recent focal point being complex spatio-temporal structures and related processes (e.g., [32,49]).

The subject of the present work is the objective detection and visualization of $3 \mathrm{D}$ frontal structures from numerical simulation output. The primary goals are facilitating further research to improve understanding of fronts and related weather systems, as well as application within forecasting. A number of studies have investigated the automated detection of 2D frontal lines from numerical simulation output, for weather forecasting, for creating climatologies, and for other applications (e.g., $[3,14,15,17])$. However, to our knowledge, no previous method exists that can detect and visualize the full 3D structure of fronts, and facilitate a rapid analysis of vertical structure, frontal zone properties, and related atmospheric processes, such as vertical motion, moisture transport and precipitation (cf. Locatelli et al. [22]). Such an approach will be beneficial because:

- Using static 2D horizontal and vertical maps and sections, as now, is clearly a sub-optimal way to picture and analyze $3 \mathrm{D}$ reality. Hewson [14] pointed out the rich complexity in vertical structures in fronts, whilst Mulqueen and Schultz [32] referenced commonly occurring "double front systems". Such aspects are contrary to the widely accepted "Norwegian" conceptual model [4]. 3D visualization opens the door to investigate these inconsistencies, in so far as "reality" is represented within state-of-the-art numerical models. This is also in the spirit of Schultz and Vaughan's recom- 
mendation [49] that traditional conceptual models be revisited.

- In operational weather forecasting, accounting for the full 3D structure of fronts, using manually constructed 2D charts, is too time consuming. Typically, the most a forecaster can achieve in the available time is analysis of one or two cross sections. We would thus like to pave the way for increased operational use of $3 \mathrm{D}$ front visualization. For example, 3D visualization can enable a forecaster to rapidly check whether a rainband relates to particularly steep or even over-turning front topography aloft.

In this article, we -a team of visualization scientists and meteorologists- propose an approach to visualize and analyze frontal structures in 3D. We contribute:

- An adaptation and extension of the 2D objective front detection method of Hewson [14] to provide the first ever visualizations of continuous 3D frontal surfaces and associated meteorological features within real-world cyclones.

- A detection scheme to identify, determine and visualize characteristics of the frontal zone associated with a frontal surface based on tracing "normal curves".

- An interactive selection scheme to isolate an "interesting" front from a complex and potentially cluttered depiction of a large region and to determine statistical information about that frontal surface, its associated frontal zone, and related processes.

- Various visualizations that relate the frontal structure to associated atmospheric processes (e.g., vertical motion, moisture transport and precipitation).

We demonstrate how our method facilitates, for example, the analysis of the vertical "extent" of a front and distinction between low-level and upper-level fronts; the horizontal breadth of a frontal zone and strength of, e.g., temperature and humidity gradients; the slope of a frontal surface, undulations on it, and their influence on vertical motion; distribution of physical parameters within a frontal zone; and the relative locations of front, jet stream, and surface pressure distribution.

Our work has been partly motivated by ongoing analysis of midlatitude cyclones and associated frontal systems observed during the atmospheric science field campaign "NAWDEX" [45], in which two of the authors were involved. This research on predictability of weather investigates how different physical processes can influence cyclone evolution. Fronts are an integral part of the NAWDEX cases and indeed of other cyclones, and rapid analysis of their 3D characteristics is required to gain improved understanding and improve future predictions.

\section{Basics AND RELATEd Work}

We first introduce a definition of atmospheric fronts and review related work on objective detection methods from the atmospheric sciences. The present article adds to the literature on visualization in meteorology, a comprehensive overview of which was recently presented in the survey by Rautenhaus et al. [36]. In other areas of visualization, surfacetype features are of importance as well, and briefly reviewed below.

\subsection{Definition of Atmospheric Fronts}

The "traditional" notion of a front as the horizontal boundary between two air masses, i.e., volumes of air with nearly coherent characteristics (e.g., [56]), dates back to the "Bergen school", about 100 years ago (e.g., [4]). The glossary of the American Meteorological Society (AMS) defines a front as "the interface or transition zone between two air masses of different density", stressing that a front almost "invariably separates air masses of different temperature" but noting that many other features may distinguish a front, including a change in wind direction or a moisture discontinuity [1]. Hewson [14] describes this interface as a "thin layer, or non-rigid slab-like region, in three-dimensional space, within which there are [...] large horizontal gradients in the thermal characteristics". Fig. 2 illustrates the concept.

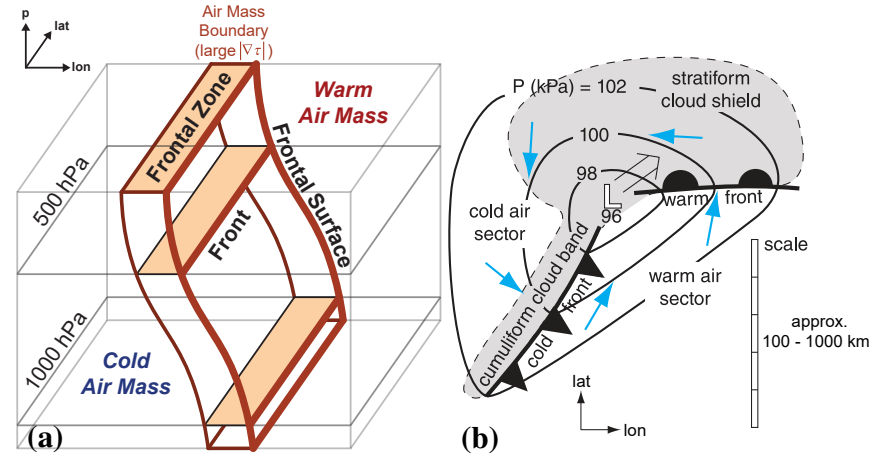

Fig. 2. (a) Illustration of a frontal surface and associated volumetric frontal zone separating warm and cold air masses in a 3D temperature field $\tau$. The frontal surface (front) is located on the warm-air side of the frontal zone. (b) Components of a typical extratropical cyclone in the Northern Hemisphere, highlighting the pivotal role of cold and warm fronts. (Adapted from [56]. Copyright 2017 R. Stull, CC-NC-SA 4.0 license.)

Important in this respect is that only the horizontal thermal gradient is considered. Although fronts commonly slope (Fig. 2a), and so also have a vertical thermal gradient associated, a more extreme class of vertical gradients also occurs in the atmosphere and for this the meteorological cause is the "opposite" of frontal processes. Such gradients are due to large-scale subsidence (commensurate with settled weather) and are known as anticyclonic inversions. Clearly one must avoid misidentifying these as fronts. Typical values for an anticyclonerelated vertical gradient are orders of magnitude greater than they are for a strong horizontal frontal gradient (e.g., $10 \mathrm{~K} / 100 \mathrm{~m}$ versus $10 \mathrm{~K} / 100 \mathrm{~km}$, respectively). Meanwhile Milionis and Davies [31] showed that inversion frequency and average inversion strength (for a UK site) are both greater in anticyclonic than in cyclonic (frontal) conditions. These two points further emphasize the need to focus on just horizontal rather than 3D gradients when identifying fronts.

In Fig. 2a, where the vertical scaling is greatly amplified relative to the horizontal, edges of the 3D frontal region are marked in brown. In the $2 \mathrm{D}$ definition, intersection of the this region with pseudo-horizontal surfaces is denoted by a "frontal zone" (or "transition zone" in the AMS definition). According to Martin [27], the length of the frontal zone is significantly greater than its breadth. Note that in this paper, we will denote the full 3D frontal layer as the frontal zone. The warm side of the frontal zone on a level is the "front"; atmospheric dynamics dictate that this is where discontinuities in other parameters, such as the wind field, should typically lie (e.g., $[14,56])$. Some authors have also considered vorticity maxima as an identifying feature, or recommended using "frontogenesis" (usually the total derivative with respect to time of the magnitude of the horizontal temperature gradient) to define frontal regions (e.g., $[42,50])$.

A number of different types of fronts are distinguished (cf. $[50,56])$; In the present paper, we consider synoptic-scale phenomena in extratropical cyclones (vs. mesoscale phenomena). Fig. $2 \mathrm{~b}$ is a snapshot of the the location of fronts in a typical mid-latitude cyclone, approximately following the "Norwegian" model dating back to the Bergen school around 1920 [4]. The model still nowadays finds application in synoptic meteorology and operational forecasting. It was extended by the "Shapiro-Keyser" conceptual model [52], which differs in the structure of the "occlusion process", the "merging" of warm and cold fronts. An overview of the literature about fronts and related conceptual models is provided by, e.g., Schultz and Vaughan [49].

\subsection{Objective Fronts}

In operational meteorology, identification and tracking of fronts are crucial, as emphasized, e.g., by Hewson [14] and Schultz and Blumen [50]. As noted in Sect. 1, 2D surface fronts are usually manually analyzed by weather forecasters. Such analyses are commonly based on many 
different parameters and inherently subjective; research has shown that different forecasters frequently recognize different fronts from the same data (e.g., [28, 43]). In this respect, Schultz and Blumen [50] pointed out that "part of the dilemma with frontal analysis is that the characteristics of fronts used for analysis are not clearly defined."

Hence, a number of studies have investigated the objective identification of frontal lines, dating back to a paper by Renard and Clarke [39] in the 1960s. Analogous to manual analysis, research focussed on two dimensions, and included application to forecasting (e.g., $[14,15])$ and to compute climatologies of the occurrence of fronts (e.g., $[3,17,47,53])$. A review of objective identification works up to the late 1990s is contained in Hewson [14]; more recent studies have also included investigation of specific processes related to the identified fronts (e.g., [16-18, 35,46]). The method by Hewson and Titley [15] is operationally run at the European Centre for Medium-Range Weather Forecasts (ECMWF) to produce products including the one shown in Fig. 1a.

Common to most published methods is the identification of a front based on a thermal parameter $\tau$ that is representative of the considered air masses. A common choice is a moist potential temperature such as wet-bulb potential temperature $\theta_{w}$ or equivalent potential temperature $\theta_{e}$, both essentially conserved under moist adiabatic ascent and hence essentially invariant to vertical motion in front vicinity. Details on these quantities can be found in meteorology textbooks (e.g., [56]). The frontal definitions referenced in Sect. 2.1 are directly applied to $\tau$; the objective identification methods seek to find regions where the magnitude $M_{\tau}=\left|\nabla_{h} \tau\right|$ of the horizontal gradient of $\tau$ is large (the frontal zone) and subsequently the warm side of this zone, i.e., essentially parts of ridge lines in the first derivative of $M_{\tau}$ (cf. [14]). Some studies, however, employ simpler criteria. The method proposed in this article is based on the method by Hewson [14,15], which can be considered representative of the current state of the art in meteorology.

\subsection{Extremal Structures in Visualization}

The existing 2D objective front detection methods extract line-type features; the method we propose generates surface-type features in 3D space. The extraction and visualization of line-type and surfacetype features is also an essential tool and ongoing research branch in flow visualization. The importance of such originates from the highdimensional nature of spatio-temporal varying flow fields -volumetric scalar and vector fields over time- and the difficulty to visually represent their inherent chaotic and turbulent structures in 3D. This requires effective visualization techniques to reduce the complexity of flow fields and leverage 3D analysis.

Related to the frontal features we extract are local extrema in ndimensional scalar fields, i.e., 2D ridge (valley) lines and 3D ridge surfaces [5,7]. 2D ridge (valley) lines are widely used in computer vision and image analysis to depict characteristic structures that exhibit a local maximum (minimum) along the transverse direction [13,21,26] For ridge surfaces, this direction is derived locally from the full 3D tensor describing the field's variation in the surrounding. Furst and Pizer [10] proposed a technique, called Marching Ridges, to obtain ridge surfaces from three-dimensional scalar fields, by tracing their transverse direction through the volume. Kindlmann et al. [20] applied ridge surfaces to visualize diffusion tensor MRI data. In flow visualization, ridges serve as an indicator and approach to determine and extract vortex core lines [55], flow separations [51], or to visualize vorticity and strain [41]. Sadlo et al. [40] used ridge detection to reveal separating regions of different flow behavior in unsteady vector fields. Peikert et al. [33] proposed a method to compute ridge lines and ridge surfaces from n-dimensional scalar fields without the explicit computation of eigenvectors from the Hessian matrix. In our scenario, where the horizontal gradient magnitude is orders of magnitudes smaller than the $3 \mathrm{D}$ gradients, ridge surface extraction does not produce meaningful structures. Even in 2D, where in our case classical ridge line detection requires a fourth-order derivative, highly fuzzy and disconnected structures can occur.

\section{Design Objectives and Method Overview}

We propose a $3 \mathrm{D}$ visualization approach that enables meteorologists to explore, for the first time, the structure of atmospheric fronts which are fundamentally 3D features, and to examine frontal characteristics and related atmospheric processes. Our design, motivated the lack of such an approach, e.g., in the ongoing NAWDEX analyses (cf. Sect. 1), targets the following: (a) Analysis of the full 3D spatial structure of frontal surfaces (to judge, e.g., spatial coherence and steepness of the surface topography) in the context of the atmospheric environment (i.e., integration of the front display with existing meteorological visualizations). (b) Analysis of atmospheric quantities at the front location and in its vicinity (e.g., vertical motion, humidity). (c) Analysis of the frontal zone associated with a frontal surface, including its spatial structure and distribution of atmospheric quantities in the zone, to focus in on regions where the physical processes that drive adverse weather are concentrated. (d) Joint analysis of frontal structures with related processes and features (e.g., jet-streams, precipitation).

To achieve these goals, we show how one can depict, in 3D, frontal surfaces and frontal zones color-coded to represent various important atmospheric quantities and related properties. Due to known skepticism in the meteorological community regarding $3 \mathrm{D}$ visualization (cf. the discussion in Rautenhaus et al. [36]), consideration of spatial perception and interactivity was deemed important. To facilitate combined visualization with further atmospheric features, we have integrated our approach into the state-of-the-art open-source meteorological visualization tool "Met.3D" [30,38]; by providing our method in an existing tool we also ease promulgation into the meteorological community.

The proposed detection method for 3D front surfaces (Sect. 4) follows the 2D approach by Hewson [14] and uses an arbitrary thermal parameter $\tau$ (cf. Sect. 2.2) selected by the user to compute feature candidate surfaces representing potential fronts. The candidates are filtered according to a number of criteria to obtain the final front surfaces. Since, to filter candidate features, the existing 2D methods discussed in Sect. 2.2 use "hard" threshold values selected based on the specific data investigated, we propose interactive adjustment of these criteria by the user to facilitate investigation of the effect of changing, e.g., the minimum strength of the thermal gradient. For visualization of the frontal zones associated with the surfaces (Sect. 5), "normal curves" are used to simultaneously display horizontal breadth of the zones, structure of the thermal gradient, and the distribution of any NWP quantity of interest. To remove clutter from a complex scene, a front feature of interest can be selected and displayed in isolation. Frontal-zone distributions of an NWP quantity of interest can be displayed for any selected front by means of a histogram. Finally, the depiction can be combined with visualizations of further features of interest, e.g., jet-stream core lines [19].

\section{Detection and Visualization of Front Surfaces}

This section describes the extraction, filtering, and visualization of $3 \mathrm{D}$ front surfaces. We introduce our method with data from a numerical simulation of an atmosphere on an "aquaplanet", a flat planet covered only by water. Details of the simulation are described by Schäfer and Voigt [44], it develops a series of cyclones that exhibit an idealized structure. One of these is selected to introduce our method with relatively "smooth" data before investigating real-world cases in Sect. 6. The aquaplanet simulation is available on a regular latitude-longitude grid with a grid spacing of $0.5^{\circ}$ (approximately $50 \mathrm{~km}$ ) in both horizontal dimensions; in the vertical 35 levels of constant pressure are available. Fig. 4a shows a horizontal map of $\theta_{w}$ of the selected region on the $925 \mathrm{hPa}$ pressure surface, illustrating the clear separation of cold air masses in the north and warm air masses in the south.

At its core, our approach closely follows the method proposed for 2D front lines by Hewson [14]. His paper provides a thorough discussion of the mathematics involved; here, we provide a brief overview of the fundamental aspects and in particular discuss its extension to 3D.

\subsection{Objective Detection of 2D Feature Candidates}

Hewson's [14] 2D method is based on detecting the "boundaries" of regions of strong gradient magnitude in a thermal parameter $\tau$, in 
(a)

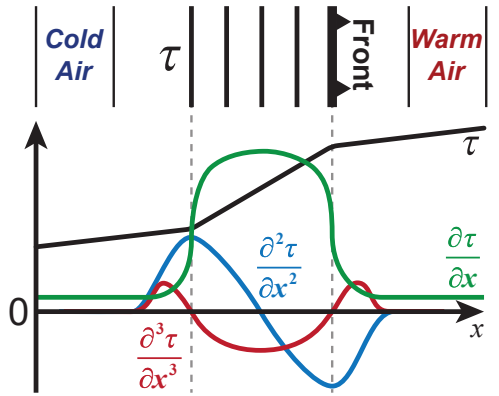

Fig. 3. Simplified 1D illustration of front detection based on a therma parameter $\tau$. (a) Schematic contour lines of $\tau$, increasing to the right, with an increased thermal gradient in the middle. (b) Profiles of $\tau$ (black) and its derivatives (color) along a straight line through the field. The front, i.e., the warm air side of the region of increased thermal gradient, is detected as a minimum in the curvature (blue) of the thermal field.

his paper taken to be the wet bulb potential temperature $\theta_{w}$. Fig. 3 illustrates the approach by assuming a simple 1D thermal gradient and a straight front geometry. In the example, $\tau$ increases linearly from left to right, the frontal line is located at the warm boundary of the frontal zone (i.e., the zone of high gradient magnitude of $\tau$ ) shown in the middle. In this case, detection of the front location is a simple 1D problem. The frontal zone is bounded by the positions at which the thermal gradient magnitude, in this case $M_{\tau}=|\partial \tau / \partial x|$, changes most rapidly. That is, the extremal points in the gradient of $M_{\tau}$, or where the third derivative of $\tau$ equals zero. In the example, the warm air side of the frontal zone, i.e., the front, is represented by the extremal point at which the thermal curvature $\partial^{2} \tau / \partial x^{2}$ is minimal.

In the general 2D case, fronts are curved and possess an along-front thermal gradient [14]. For this case, Hewson [14] formulates the "front locating equation" $[\mathrm{L}]$ as

$$
L_{\tau} \equiv \frac{\partial\left(\left|\nabla_{h}\right| \nabla_{h} \tau||\right)_{s}}{\partial s}=0, \quad \text { with } \hat{s}= \pm \frac{\nabla_{h}\left|\nabla_{h} \tau\right|}{\left|\nabla_{h}\right| \nabla_{h} \tau||}
$$

where $\nabla_{h}$ denotes the horizontal gradient and $\hat{s}$ represents a unit axis (i.e., possessing only an orientation instead of a direction as a unit vector would) oriented along the gradient of the thermal gradient magnitude $M_{\tau}=\left|\nabla_{h} \tau\right|$. That is, essentially the ridge lines in the 2D height field represented by $\left|\nabla_{h} M_{\tau}\right|$ are sought. While classical ridge line detection [8] requires even a fourth-order derivative on $\tau$ and evaluation of the Hessian matrix, a simpler computational scheme was suggested by Hewson [14]. In short, his scheme derives a five-point mean axis $\hat{s}$ from the horizontal grid points surrounding a considered grid point, then evaluates the locating equation [L] by means of computing the "along-vector divergence" of $\nabla_{h} M_{\tau}$. Hewson [14] shows that the vector field $\nabla_{h} M_{\tau}$ possesses zero divergence along the frontal line; the notion of "along-vector divergence" is introduced for numerical stability, here simply all vectors at grid points employed for computation of the divergence are resolved into the direction of $\hat{s}$. Due to space limitations, we refer to Hewson [14] for a thorough description. In our approach, we follow the Hewson scheme to achieve consistency with 2D products that are operationally produced at ECMWF (cf. Fig. 1). Fig. 4a shows 2D feature candidates obtained for the idealized aquaplanet case.

\subsection{Extension to 3D}

The critical question that arises when raising the approach to three dimensions is whether vertical contributions to the gradient need to be considered. The meteorological definitions reviewed in Sect. 2 clearly only consider the horizontal gradient of $\tau$. As discussed there this is primarily to avoid mis-representation of non-frontal features as fronts. Inspection of a vertical section through a frontal zone (Fig. 5a) may suggest that the locating equation $[\mathrm{L}]$ should be evaluated in the direction of a three-dimensional axis $\hat{s}$. Note, however, that in all depictions the vertical scale is massively exaggerated, with horizontal gradient magnitudes $\nabla_{h} M_{\tau}$ being approximately three orders of magnitude smaller than 3D gradients (Fig. 5b and c), which would again result in significantly different (often non-frontal) features being detected if a 3D gradient were used. We hence use only horizontal derivatives in the computation of our frontal features. A problem we encountered with this approach, however, is that some frontal surfaces may exhibit "holes" that appear in cases of locally reduced horizontal gradients of $M_{\tau}$ (an example is arrowed in Fig. 5). In the case shown, the definition of a front as a horizontal boundary between two air masses is not fulfilled. However, intuition may suggest that nevertheless a frontal surface should be drawn in this case; an issue that may require further investigations and possible re-assessments of the employed frontal definitions in the future.

Our approach to detect 3D candidates is hence: (a) At each grid point of the data, the locating equation $[\mathrm{L}]$ is evaluated as proposed by Hewson [14] to obtain a 3D scalar field of [L]. (b) 3D contouring methods (ray-casting, e.g., [9], or Marching Cubes [25]) are used to obtain raw candidate features that subsequently need to be filtered to obtain the desired frontal features. Fig. $4 \mathrm{~b}$ shows the candidate surface features thus obtained.

\subsection{Filtering of 3D Feature Candidates}

The obtained candidate features need to be filtered to obtain those that actually represent frontal surfaces. Hewson [14] filters 2D candidates by means of two distinguishing characteristics: the curvature of the thermal parameter field, and the magnitude of the thermal gradient in the vicinity of the candidate feature as a measure of the frontal strength.

To obtain features at the warm-air side boundary of the frontal zone, all candidate features at the cold-side boundary are removed by keeping only the set of candidate vertices at which the curvature is negative. For this purpose, Renard and Clarke [39] introduced the "thermal front parameter" (TFP) as a negated curvature:

$$
T F P_{\tau} \equiv-\nabla_{h}\left|\nabla_{h} \tau\right| \cdot \frac{\nabla_{h} \tau}{\left|\nabla_{h} \tau\right|}>K_{1}
$$

where $K_{1}$ is a user-defined threshold; it is required to be at least zero to obtain features on the warm air side of the frontal zone (cf. the blue line in Fig. 3 and note the negation). Hewson [14] sets $K_{1}$ to a small positive value to eliminate spurious features (criterion [M1] in his paper).

To require detected fronts to represent a minimum specified strength in terms of thermal gradient, Hewson [14] uses an additional filter criterion that estimates frontal strength by approximating the local magnitude of the gradient of $\tau$ on the cold side of the considered candidate feature point. Candidates are eliminated if they do not fulfill

$$
S_{\tau} \equiv\left|\nabla_{h} \tau\right|>K_{2}
$$

where $K_{2}$ again is a user-defined threshold (criterion [M2] in [14]). In the approach by Hewson [14], values $K_{1}$ and $K_{2}$ are found specific to the employed NWP data and vertical levels. Candidate features are filtered "hard", i.e., all candidate points that do not meet the specified criteria are eliminated entirely. Fig. $4 \mathrm{c}$ illustrates the 2D case.

For detection of 3D frontal features, these filter criteria pose several disadvantages. First, estimation of frontal strength based on thermal gradient magnitude near the candidate feature is not necessarily representative of the gradient inside the frontal zone. Second, replacing a "hard" by a "soft" filtering, i.e., not discarding candidates completely but mapping the criterion to opacity, can help to smoothly "fade", e.g., regions of strong frontal strength to regions of weak strength, thus keeping additional information in the visualization. Third, suitable values of $K_{1}$ and $K_{2}$ may depend on the data or the specific structure in which the user is interested. In an interactive application as envisaged in our work, interactive adjustment is hence required. Fourth, filtering can be improved by considering further characteristics of the frontal zone. For instance, Hewson and Titley [15] suggested to eliminate fronts resulting primarily from moisture and not temperature gradients by adding a frontal strength criterion based on (dry) potential temperature.

Our approach improves upon all mentioned aspects. Contrary to estimating the strength of a front by evaluating Eq. 3 only by local 

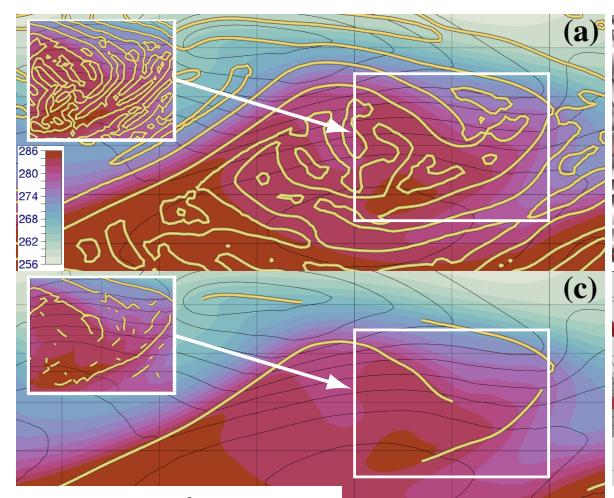

$K_{1}=0.48 \mathrm{~K} /(100 \mathrm{~km})^{2} ; K_{2}=1 \mathrm{~K} / 100 \mathrm{~km}$

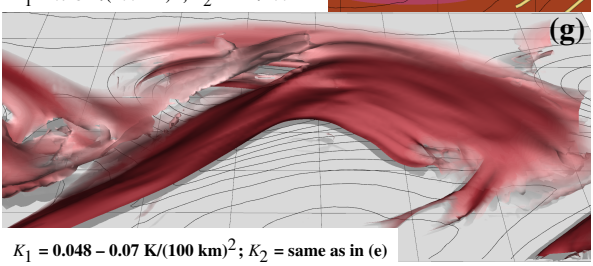

(c)

)
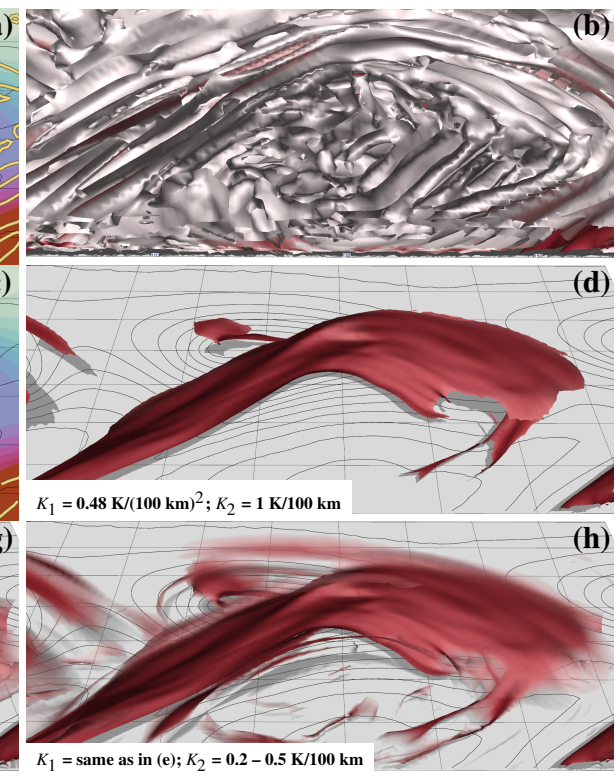

(d)

(h)

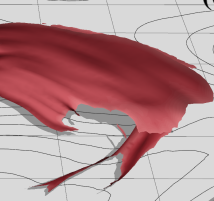

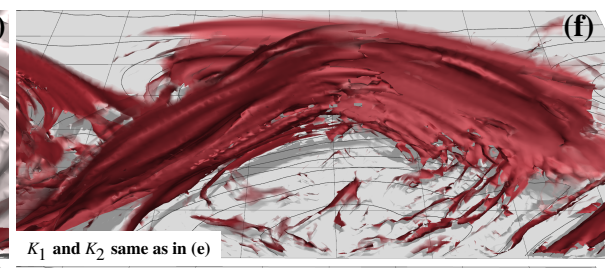
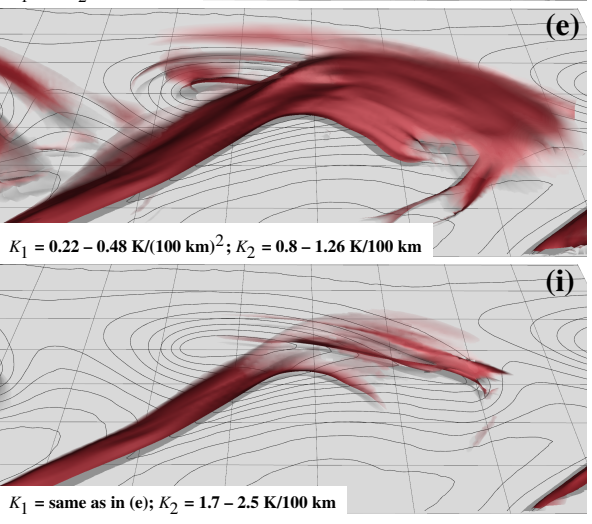

(i)

Fig. 4. 2D and 3D objective detection and filtering of front surface features (idealized aquaplanet dataset). Note non-sequential order of images. (a) Horizontal section at $925 \mathrm{hPa}$, showing $\theta_{w}$ (K, color), geopotential height (black contours), and 2D feature candidates (following [14], yellow lines). Gaussian smoothing with a distance of $100 \mathrm{~km}$ has been applied to $\theta_{w}$. Inset compares detected features candidates if no smoothing is applied. (b) 3D feature candidates for the same case. (c) "Hard" filtering with the listed thresholds for $K_{1}$ and $K_{2}$ applied to the feature candidates in (a). Inset compares the unsmoothed field as in (a). (d) "Hard" filtering applied to the 3D feature candidates in (b). Black contours shows surface pressure. (e) As (d) but with "soft" filtered features. The listed range of $K_{1}$ and $K_{2}$ has been linearly mapped to opacity [0-1]. (f) The same as (e) but with decreased smoothing of $\theta_{w}(25 \mathrm{~km}$ compared to $100 \mathrm{~km}$ in (e)). Note the increased number of small-scale features. (g-i) Same as (e) but with different settings of the filter values $K_{1}$ and $K_{2}$ to demonstrate the sensitivity of detected front surfaces on these values. Detected features become larger for relaxed $(\mathrm{g}, \mathrm{h})$ filter values; more restrictive values allow focus on the stronger parts of a front (i).

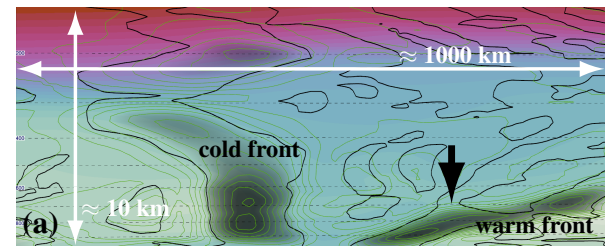

(b)

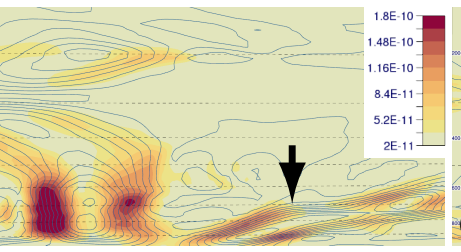

(c)

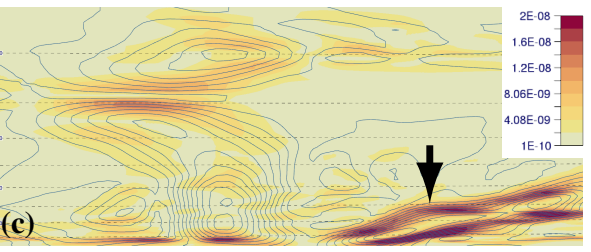

Fig. 5. Considering vertical contributions of derivatives to identify the boundaries of the zone of high thermal gradient leads to undesired features. Shown are vertical sections of (a) $\theta_{w}$ (K, color as in Fig. 4a), green contours show thermal gradient $M_{\tau}=\left|\nabla_{h} \tau\right|$, regions of large $M_{\tau}$ are shaded in black. Black contours denote $L_{\tau}=0$, i.e., sections through the feature candidates. Note the apparent "hole" in the warm front (arrow), where the $L_{\tau}$ contour moves upward. (b) Magnitude of horizontal-only gradient $\left|\nabla_{h}\right| M_{\tau}||\left(\mathrm{K} \mathrm{m}^{-2}\right.$, color), blue contours show $M_{\tau}$. The feature candidates in (a) are essentially ridges in $\left|\nabla_{h}\right| M_{\tau}||$. In the region of the "hole" $\left|\nabla_{h}\right| M_{\tau}||$ is weak. (c) When using the full 3D gradient $|\nabla| M_{\tau}||$, the "hole" is "filled", however, fundamentally different features are detected. E.g., the cold front (cf. (a)) vanishes. Note the much stronger gradient magnitude compared to (b).

approximation, we define as an estimate of frontal strength the average thermal gradient along a curved path through the frontal zone from the warm to the cold-air side. Here, we apply the concept of "normal curves" to traverse the frontal zone. Normal curves are traced through a scalar field following its gradient direction. They were used by Paffelmoser et al. [34] to measure the spatial distance between two isosurfaces, Rautenhaus et al. [38] used them to visualize the interior structure of isosurfaces. Fig. 6a illustrates the approach. A "straight line normal" can not always represent a path across the breadth of the frontal zone, particularly if a front is highly curved as is often the case when it is particularly active. A normal curve, on the other hand, runs everywhere parallel to the thermal gradient that we are interested in averaging across the zone. Again, only horizontal gradients are considered as by definition (cf. Sect. 2.1) the horizontal breadth of the zone is the quantity of interest, so all normal curves lie fully within "horizontal" planes (that is planes that accord with the vertical axis definition). Eq. 3 then becomes

$$
\left.S_{\tau}\right|_{\text {frontal zone }} \equiv \int_{N C}\left|\nabla_{h} \tau\right| d s>K_{2}
$$

Traversal is stopped upon hitting the "cold-side" boundary, where

\section{$L_{\tau}=0\left(L_{\tau}\right.$ is always negative within the frontal zone; cf. Fig. 3$)$.}

To construct the frontal surface visualizations, we implemented two rendering approaches. A ray-casting-based approach performs all filtering computations including normal curve integration on the GPU upon hitting a candidate surface (i.e., where $L_{\tau}=0$ ); filtering, colorand opacity-mapping is performed per-pixel. A polygonal approach extracts surface mesh geometry using Marching Cubes, and performs filtering, color- and opacity-mapping per vertex. Rendering employs order-independent transparency. The approaches differ in particular with respect to performance. While the ray-casting approach requires more rendering time (for the cases considered here up to a few seconds on an NVidia Geforce GTX 970), the polygonal approach requires preprocessing time on the order of 10 seconds but subsequently facilitates adjustment of filtering criteria at interactive framerates.

Fig. 4d and e illustrates the difference of "hard" versus "soft" filtering for 3D frontal surfaces. "Soft" filtering is implemented with transfer functions that map $K_{1}$ and $K_{2}$ to opacity. This facilitates an interactive, user-guided filtering process (for polygonal rendering); the mapping of $K_{i}$ to opacity can be quickly adjusted, and thus the sensitivity of the detected surfaces on, e.g., frontal strength, estimated. Fig. 4g-i shows 


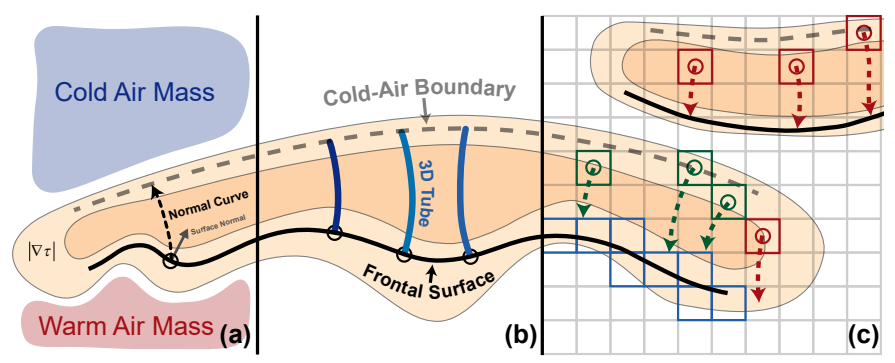

Fig. 6. Normal curves are used for multiple aspects of our approach. Shown is a schematic region of high thermal gradient (orange colors), with a frontal surface detected on its warm air side (thick black line). (a) Normal curves (dashed arrow) cast from the frontal surface through the frontal zone are used to obtain curve-integrated quantities including frontal strength. (b) For depiction of the frontal zone, normal curves are rendered as tubes. (c) A candidate grid cell belongs to the frontal zone associated with a selected frontal surface (cf. Fig. 8) if a "backward" normal curve started from the cell center intersects with the selected frontal surface (green cells and curves). Cells with curves intersecting with none or a different surface are discarded (red cells and curves).

examples of how the detected surfaces change when the transfer functions are changed. Hewson [14], specific to the data used in his study, suggests values for $K_{1}=0.3 \mathrm{~K} /(100 \mathrm{~km})^{2}$ and $K_{2}=1.35 \mathrm{~K} / 100 \mathrm{~km}$; in Fig. 4, values are of similar order but vary as noted.

\subsection{Data Smoothing}

Increasing horizontal resolution of NWP models (cf. [36]) has in recent years enabled the models to resolve increasingly smaller details of the atmosphere. While this for many application is a valuable property, for the detection of synoptic-scale fronts removal of small-scale gradients and variability in the data is desirable to obtain smooth features that represent the large-scale features well. Jenkner et al. [17], as well as others, used a simple smoothing filter assuming equally distributed grid points in terms of geometric distance. To account for grid points in a regular latitude-longitude grid to be closer together near the poles, we instead use a 2D Gaussian kernel to smooth, under consideration of all surrounding grid points, with a user-defined geometric distance. This approach removes sensitivity of our method to the grid resolution of the underlying data; instead, sensitivity to the smoothing distance is present. Fig. $4 \mathrm{e}$ and $\mathrm{f}$ illustrates the sensitivity of detected 3D features to changing the standard deviation of the kernel smoothing the $\theta_{w}$ field (Fig. $4 \mathrm{a}$ and $\mathrm{b}$ for $2 \mathrm{D}$ ); as expected larger smoothing distances eliminate small-scale features. Typical length scales for the fronts of interest in this study are on the order of 100 to $1000 \mathrm{~km}$ (cf. Sect. 2.1 and Fig. 2), smoothing should be performed accordingly. For the cases presented in this paper, we selected a standard deviation of $100 \mathrm{~km}$. Note that smoothing affects the settings of the filtering parameters $K_{1}$ and $K_{2}$, since with increased smoothing the thermal gradients are weakened.

\subsection{Visualization of Front Surfaces and Related Processes}

The detected frontal surfaces are rendered in Met.3D [38] and can be combined with existing displays of the system, e.g., horizontal maps and vertical cross-sections. Properties of the front and surrounding atmosphere (e.g., frontal strength and atmospheric variables interpolated to the frontal position) can be color-mapped onto the surfaces (cf. the tasks in Sect. 3). Fig. 7 provides examples. Note the use of shadows and movable vertical axes (Fig. 7a) to improve spatial perception, and combination with movable $2 \mathrm{D}$ sections through the atmosphere (Fig. 7c; cf. [38] for these functions). Color scales use the perceptional linear HCL color space [54] to conform with common visualization functionality in Met.3D (e.g., cf. the case study in [37]). Fronts are classified as cold or warm fronts, depending on whether the local wind advects temperature change towards cold or warm air, following Hewson [14]: $A_{\tau}=-V \cdot \nabla_{h} \tau$, where $V$ denotes horizontal wind; Fig. 8a shows an example. Furthermore, our approach facilitates computation and display of the frontal slope, important to study, e.g., relationships between surface topography and precipitation (cf. [22]). Combination with visualizations of further atmospheric features including air parcel trajectories [37] and jet-stream core lines [19] is also readily available. The latter will be demonstrated in the Sect. 6 .

\section{AnALYSIS OF Frontal ZONES AND INDIVIDUAL FrontS}

In addition to visualization of frontal surfaces, another unique feature of our approach is the interactive visual analysis of frontal zones associated with a frontal surface. The proposed techniques enable users to analyze the horizontal breadth of a frontal zone, as well as the distribution of atmospheric variables within (cf. task (c) in Sect. 3). To reduce clutter and to facilitate examination of an individual feature of interest, single front features can be selected and isolated.

\subsection{Normal-Curve-Based Visualization}

As described in Sect. 4.3, we evaluate frontal strength at a frontal surface point by integrating along a "normal curve" in the thermal gradient field. It is straightforward to use this approach to obtain further frontal-zone-averaged quantities, e.g., humidity. Values obtained can be color-mapped onto the frontal surface to display properties of the zone associated with the surface. Also, curve length can be used to provide color-coding of the zone's horizontal breadth.

Normal curves also offer a way to directly depict the frontal zone and the structure of its gradient field. Fig. $6 \mathrm{~b}$ illustrates the concept, showing a set of curves that start on the frontal surface as 3D tubes. We follow Rautenhaus et al. [38] to generate seed points by computing the intersections between rays parallel to the coordinate axes and the frontal surface (see [38, Sect. 4.4] for details). For our application, an additional check is required to account for the filter criteria (cf. Sect. 4.3); potential seed points at which the identified front surface has been made transparent by the filtering transfer functions are eliminated. The density of displayed curves can be controlled by the user (cf. [38]).

Fig. 7b and c shows an example. The normal curves act as an indicator of the breadth of the frontal zone. Simultaneously, the structure of the thermal gradient field inside the zone is conveyed by the shape of the curves (cf. [38, Sect. 3.4]). Rendering the normal curves as 3D tubes also facilitates color-coding of scalar measures including gradient magnitude itself (cf. Fig. 7b and c) or arbitrary atmospheric quantities.

\subsection{Selection of Individual Fronts}

The simultaneous visualization of all front/frontal zone features detected in a scene can result in cluttered displays. We hence propose a mechanism to select and isolate a single front feature of interest for inspection - see Fig. 8. Picking is realized by casting a ray from the virtual camera into the scene through the selected pixel, and computing the first intersection of that ray with a computed frontal surface. At the intersection point, we determine the eight surrounding grid points (i.e., a "root" grid cell) in the 3D data grid, extract the local geometry of the picked surface using Marching Cubes on the front locator $L_{\tau}$, and compute the front type (cold or warm, cf. Sect. 4.5). From the root cell, region-growing is used to iteratively identify all grid cells and front geometry that belong to the picked surface. To add a neighboring cell or surface triangle, we require adjacent triangles to form a watertight mesh and to belong to the same front type. Also, filtering criteria have to be observed; parts of the surface that are transparent in the rendered representation of the front are discarded. The approach is continued until no further cells need to be evaluated.

\subsection{Statistical Properties of a Frontal Zone}

To query statistical properties of a frontal zone (e.g., the distribution of an atmospheric parameter within the zone), a volumetric representation of the zone is required (i.e., a list of all grid cells that are part of the zone). The geometric representation of a selected frontal surface obtained in Sect. 5.2 can be used to approximately determine the volume that is enclosed by the corresponding frontal zone. Fig. 6c illustrates the approach: First, all grid cells intersected by the frontal surface are determined (blue cells in Fig. 6c). Next, a bounding box enclosing these cells is generated, which subsequently is enlarged to ensure that surrounding grid cells that may belong to the frontal zone are included. 

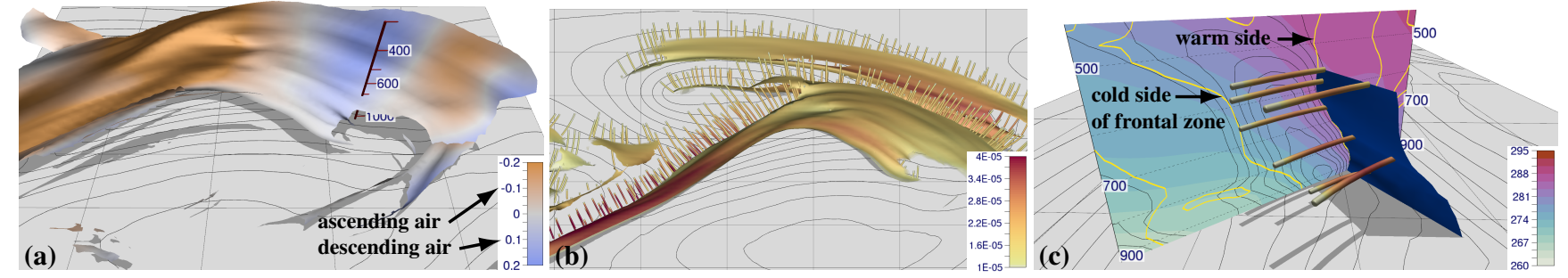

Fig. 7. Visualization of front parameters and frontal zone. (a) Vertical velocity in $\left(\mathrm{Pa} \mathrm{s}^{-1}\right)$ as an example of an atmospheric quantity color-mapped onto the frontal surface. Note the ascending motion in particular along the left part of the front (negative values indicate ascent). (b) Normal curves rendered as tubes to visualize the frontal zone. Curves are color-coded with the magnitude of the thermal gradient. Black contour lines represent surface pressure. (c) Vertical section showing $\theta_{w}$ (K, color), thermal gradient (black contour lines) and the zero-isocontours (yellow) of the locator field, which bound potential frontal zones. Note how the normal curves extend horizontally from the frontal surface throughout the frontal zone; integration is stopped upon hitting the locator "contour" on the cold side of the zone.
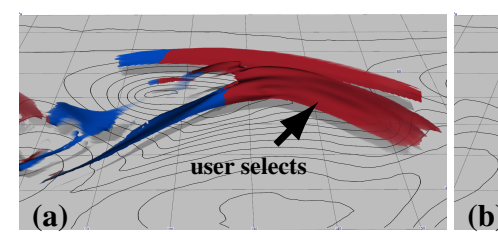

(b)
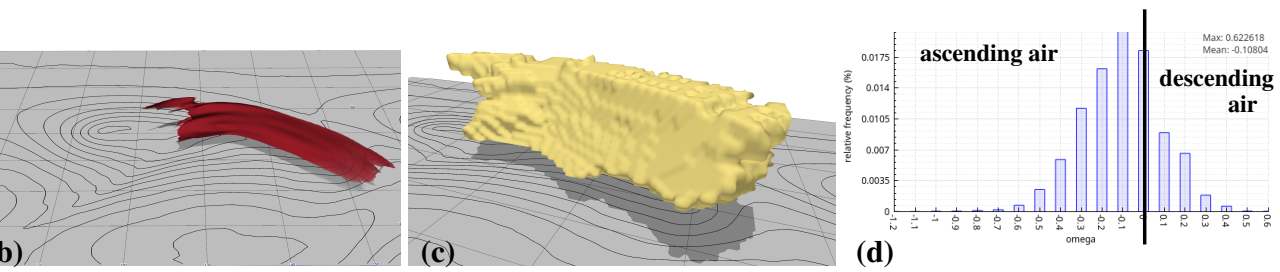

Fig. 8. Interactive selection of a front enables the user to visualize and analyze features of that particular front on its own. (a) The fronts are colored by their classification as cold (blue) and warm (red) front. The user selects the warm front, which (b) appears isolated. (c) Frontal zone detection (cf. Fig. 6c) identifies the displayed volume as the frontal zone of the selected warm front. (d) Distribution of atmospheric quantities in the frontal zone can be displayed by means of a histogram. The example shows vertical wind velocity $\omega\left(\mathrm{Pa} \mathrm{s}^{-1}\right)$.

Enlargement is done via a user-defined distance large enough to cover the scales of interest (cf. Fig. 2, for the study at hand features of sizes of order $100 \mathrm{~km}$ to $1000 \mathrm{~km}$ are of interest, a value we found suitable for the cases investigated here is $300 \mathrm{~km}$ ). For each grid cell in the bounding box, we evaluate $L_{\tau}$ (Eq. 1) and keep those within the thermal gradient zone $\left(L_{\tau}<0\right)$ and those that intersect with the cold-side boundary $\left(L_{\tau}=0\right)$ as potential candidates. Since candidate cells in the bounding box can also belong to a different front feature in the vicinity of the selected front, correspondence of the candidate cell with the frontal zone associated with the selected surface needs to be confirmed. Here, "backward" normal curves prove useful. As shown in Fig. 6c, a normal curve is traversed from the center of each candidate grid cell into the direction of the warm-side air (i.e., towards the frontal surface). If the normal curve intersects the frontal surface, the candidate grid cell belongs to the frontal zone of interest. Curves not intersecting the frontal surface are terminated as soon as they leave the bounding box.

Fig. $8 \mathrm{c}$ shows the volume thus identified for the selected warm front in Fig. 8b. It is now straightforward to compute, e.g., histograms of data values within the frontal zone. Fig. 8d shows an example: A distribution of vertical velocity has been queried, revealing prevailing upward motion (i.e., negative velocities) in the frontal zone.

\section{Results: Case Study and User Feedback}

To demonstrate the value of our method, this section discusses first investigations of the 3D frontal structure of two mid-latitude cyclones that occurred during the 2016 North Atlantic Waveguide and Downstream Impact Experiment (NAWDEX, [45]), an atmospheric research field campaign in which two of the authors were involved. The analysis of the 3D structure of the observed cyclones is a major focus of the -at the time of writing ongoing- data analysis activities of NAWDEX (cf. [45]). Here, we consider the systems "Vladiana" and "Walpurga" that both crossed the North Atlantic in late September 2016. We use analysis data (i.e., the initial conditions of subsequent forecasts and thus the "best estimate" of the atmospheric situation at the considered time) obtained from ECMWF; all figures are produced from data on a regular latitude-longitude grid with a grid spacing of $0.5^{\circ}$, using 137 terrain-following model levels in the vertical. Gaussian smoothing has been applied to $\theta_{w}$ with a horizontal standard deviation of $100 \mathrm{~km}$.

\subsection{Cyclone "Vladiana"}

Cyclone Vladiana preceded the extratropical transition of Tropical Cyclone "Karl", aspects of which were described by Kern et al. [19]. Our focus is on 00:00 UTC 23 September 2016, a time at which, after rapid intensification, Vladiana had evolved to maturity. Our initial objective is to compare its structure with idealized conceptual models and to investigate structural details of the fronts. Next, we show how the visualized $3 \mathrm{D}$ fronts facilitate investigation of the cyclone structure in a way not possible with classical front analysis at single levels.

Fig. 1 shows the operational objectively detected 2D fronts from ECMWF (Fig. 1a) and the 3D fronts from our method; Fig. 9a shows the detected 3D fronts classified as cold and warm fronts. As expected, the cold and warm 3D fronts show good agreement with the ECMWF surface fronts, but 3D visualization adds valuable insight into vertical extent and structure. The warm front appears as a downstream tilted, curved feature (red colors) on the eastern flank of the cyclone that separates northeastward moving warm air southwest of the front from westward moving cold air to its north. The westernmost part of this frontal surface is classified as a cold front, due to northerly winds on the rear side of the cyclone bringing cold air southward. At the cyclone center the lateral position of the meeting point of cold and warm fronts varies only slightly in the vertical, suggesting little tilt with height in the cyclone's frontal signature, which is probably symptomatic of a mature cyclone. Such aspects cannot be seen on a 2D front chart. Almost perpendicular to the warm front, the main cold front (blue colored) extends southwestward towards the US east coast. Several small red areas (detected warm front character) within the large cold front appear when the predominately southwesterly flow that crosses the frontal surface at small angles locally advects warm air in the direction of the cold air. When near the earth's surface these signify frontal waves or anti-waves [14] - note the orange spots on Fig. 1a. When higher up they denote upper-level-only equivalents of these [2]. In both cases a change in surface weather can be associated.

Fig. $1 \mathrm{~b}$ and $\mathrm{c}$ and Fig. 9b and c show selected key meteorological parameters color-mapped onto the detected fronts, providing insight into atmospheric processes along the frontal features that could not be inferred from the existing 2D approaches. Pressure elevation (Fig. 1c) at a glance shows that the cold front as well as the northern part of the 

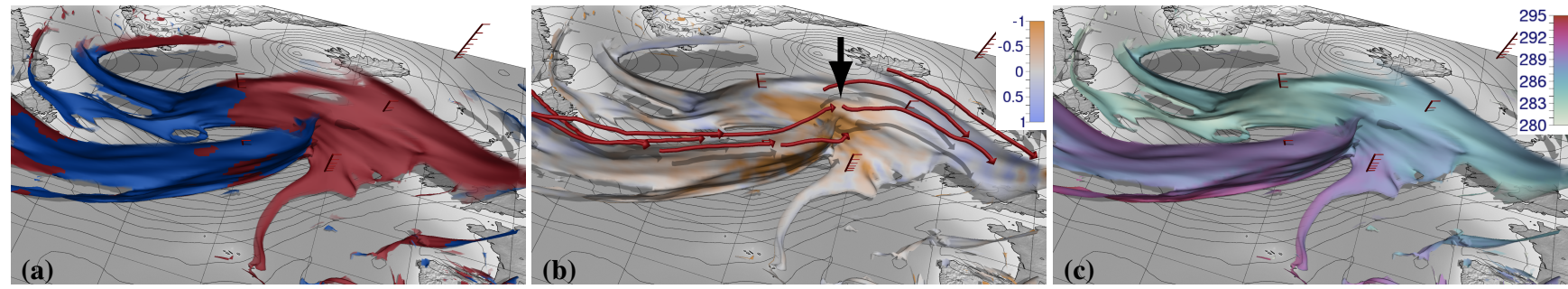

Fig. 9. Fronts of cyclone "Vladiana", at 00:00 UTC 23 September 2016. (a) Cold (blue) and warm (red) fronts. (b) Vertical wind velocity at the frontal surface ( $\mathrm{hPa} \mathrm{s}^{-1}$, negative values denote ascent). Note stronger ascent near the triple point (arrow). Red lines show jet-stream core lines detected as in Kern et al. [19]. (c) Wet bulb potential temperature $\theta_{w}(\mathrm{~K})$. Note almost constant values on each frontal surface.
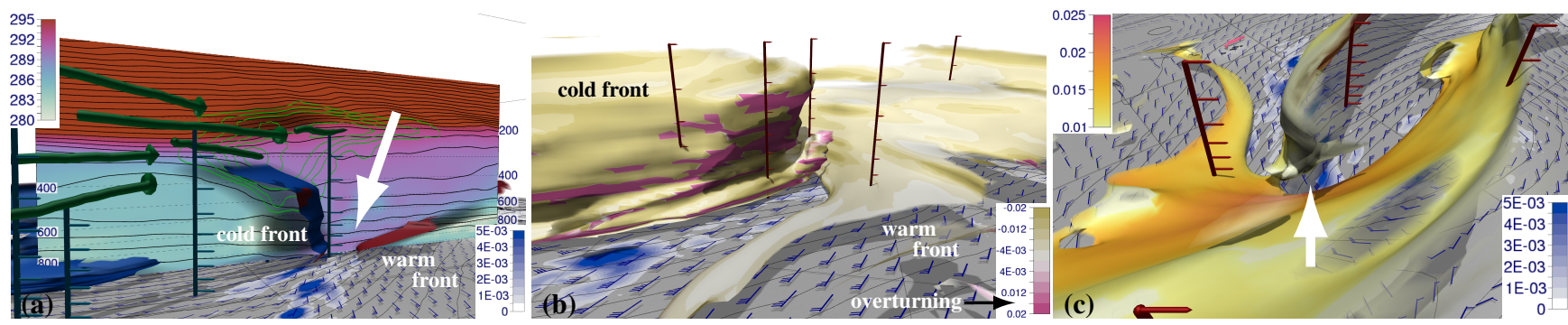

Fig. 10. Details of the fronts of "Vladiana", at 00:00 UTC 23 September 2016. (a) Vertical section through cold and warm front, showing $\theta_{w}$ (K, color), the $\theta_{w}$ gradient (black shading), contours of potential temperature (grey) and wind speed (green) and jet-stream core lines as in Fig. $9 \mathrm{~b}$. Blue ground surface shading shows precipitation $\left(\mathrm{m} \mathrm{h}^{-1}\right)$. The arrow points to the warm sector. Note the jet-stream above the cold front. (b) Color mapping of frontal slope (ratio vertical to horizontal). Note the larger slopes for the cold front than for the warm front. The cold front even exhibits some overturning (lilac). (c) Looking southwestwards; "frontal fracture" between cold and warm front extends through much of the troposphere (arrow).

warm front are deep features that extend into the upper troposphere (above $400 \mathrm{hPa}$ ). The southern part of the warm front, however, appears vertically shallow at lower levels. Air masses tend to be displaced vertically in the vicinity of the frontal zones, as the fronts themselves propagate. Often vertical velocity magnitude reflects frontal slope [22]. Indeed here we generally see higher vertical velocities where there are steeper slopes, such as near the triple point (Fig. 9b). The ascent adiabatically cools the air until saturation is reached and clouds and precipitation form. Vertical velocities are also an atmospheric response to broadscale dynamical forcing; for example the "left exit" and "right entrance" regions beneath upper level jets commonly have large upward motion associated. By adding jet cores to such plots the interplay between features can be demonstrated in new ways (Fig. 9b). The conservation of wet bulb potential temperature $\theta_{w}$ is confirmed by almost constant values on the various frontal surfaces, shown in Fig. 9c. The different colors on the different fronts show that the warm airmass associated with each has a different intrinsic $\theta_{w}$.

Fig. 10a shows a front-crossing vertical section of $\theta_{w}$, along with the detected cold and warm fronts. Vladiana's warm sector (arrowed) appears clearly as an air mass with increased $\theta_{w}$. Atmospheric dynamics tend to mean that zones of strong horizontal temperature gradients are accompanied by vertically increasing wind speeds, leading to high level jet streams (cf. [19]). The depiction in Fig. 10a shows jet cores above the cold front (cf. [52]).

The Norwegian conceptual model [4] describes the slope of typical cold fronts to be steeper (about $1 \mathrm{~km}$ vertical rise over a horizontal distance of $100 \mathrm{~km}$ ) than that of typical warm fronts $(1 \mathrm{~km}$ over $300 \mathrm{~km})$. This idealized picture is generally reflected in Fig. 10b. However, we observe a large variability in frontal slope in particular in the cold front, where overturning is present locally. This may relate to local instability causing enhanced convective precipitation (cf. [22]). An investigation of vertical instability along frontal structures is one of the scientific aims of NAWDEX; future work will employ our method to improve interpretation of campaign observations in this regard.

Our 3D visualizations provide an effective means for comparison of cyclone types with conceptual models and associated cyclone characteristics. For example, the Norwegian and Shapiro-Keyser conceptual models differ with respect to the occlusion process, i.e., the merging of cold and warm fronts (cf. [49]). The Norwegian model describes the cold front to "catch up" with the warm front; a "triple point" is formed with an elevated occluded front extending to the cyclone center. In contrast, Shapiro and Keyser [52] describe a "frontal fracture" with the cold front being detached and almost perpendicular to a strong warm front north of it. Fig. 10c indicates very clearly a detachment, through depth, of the cold and warm fronts, which seems to suggests a Norwegian cyclone. However, care is needed in interpretation as at the base of an occlusion warm air has conceptually similar characteristics to the ubiquitous anticyclonic inversions, which we have tried to eliminate from our analysis. This may be an area for future work.

To gain further insight into the characteristics of the cold front, it is selected and the frontal zone is visualized using normal curves (Fig. 11a and b). Fig. $11 \mathrm{~b}$ shows vertical velocity mapped to normal curves. As expected from Fig. 9b, ascent is represented close to the frontal surface. Our depiction shows how far this ascent extends horizontally into the frontal zone; on the back side descent is present, whilst closer to the cyclone center (left side of the figure), descent is stronger and closer to the frontal surface. The different breadths of the regions of ascent may relate to different breadths of rainbands. Frontal zone statistics become useful to obtain an estimate of the $\theta_{w}$ distribution within. As visible in Fig. $11 \mathrm{c}, \theta_{w}$ values are smaller than the values mapped onto the front in Fig. 9c, as expected from the front definition.

\subsection{Cyclone "Walpurga"}

Cyclone Walpurga, the fifth system in September 2016 observed during NAWDEX, had reached southern Scandinavia at 12:00 UTC 29 September 2016. Its long cold front extended from southern Sweden over Denmark, northwestern France to the Azores. Fig. 12 shows the operational 2D fronts product from ECMWF, along with our 3D visualization. We consider an interesting feature that immediately is visible to the user in the 3D depiction: In the southernmost part of the front, the ECMWF detection shows a secondary cold front upstream of the cold front, seemingly detached and independent from the latter. The 3D depiction, however, shows that both fronts are actually connected at upper levels in the east and belong to the same structure. The primary front appears 

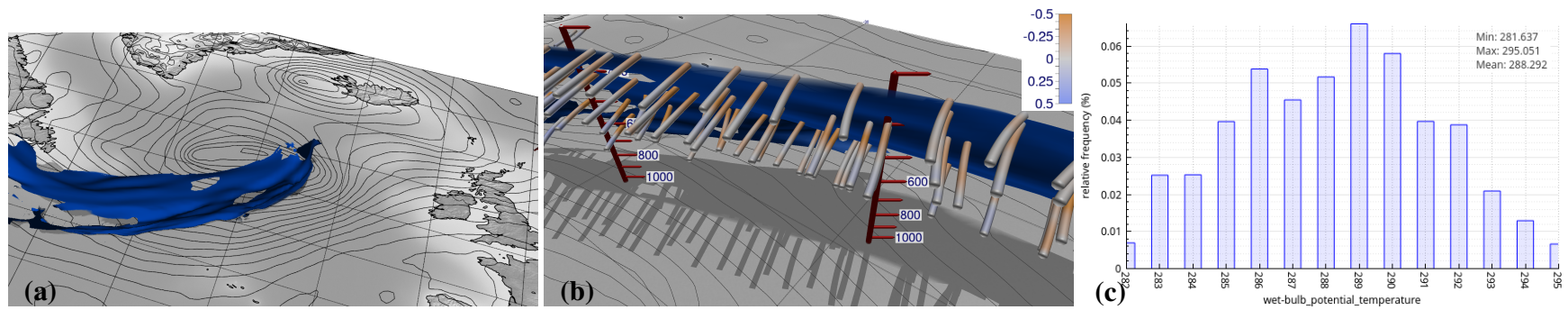

Fig. 11. The main cold front of "Vladiana" is investigated. (a) Selection of the cold front isolates the feature. (b) Normal curves colored by vertical velocity show ascending air close to the frontal surface (cf. Fig. 9b); at the rear side of the frontal zone descending air prevails. (c) Distribution of $\theta_{w}$ in the frontal zone. As expected, the values depicted on the frontal surface in Fig. 9c are on the warm side of the distribution.

to be very shallow (mostly below $900 \mathrm{hPa}$ ) whereas the trailing front is a deep feature vertically extending into the mid troposphere. This may be a case of a "double front" system as recently discussed by Mulqueen and Schultz [32]. While Mulqueen and Schultz only considered 2D surface maps, our method provides a means for a thorough systematic investigation of multiple-front cyclones. Only a full 3D perspective can clarify whether double fronts observed at the surface are individual frontal systems or interconnected.

\subsection{Domain Expert Feedback}

Domain experts, active in weather forecasting and meteorological research, were provided with some static 3D front images from this paper, and were asked to provide feedback; about 20 replied, from Europe and North America. In regard to potential use as a research tool the response overall was very positive (one said "this is really amazing!"). For real-time forecasting applications the response was also positive, though a few were concerned about speed of operation and usability. Meteorological training and media outlets were also mentioned as potential application areas.

Many indicated that tailored 3D front products could assist with aviation forecasting, specifically for major hazards like icing and "embedded convection", that usually occur within frontal cloud. Also cited were turbulence prediction, the "sting jet" extreme wind phenomena and snowbands. In research and forecasting many were interested in frontal slope, and 3D front characterization (ana- and kata-, frontoge-
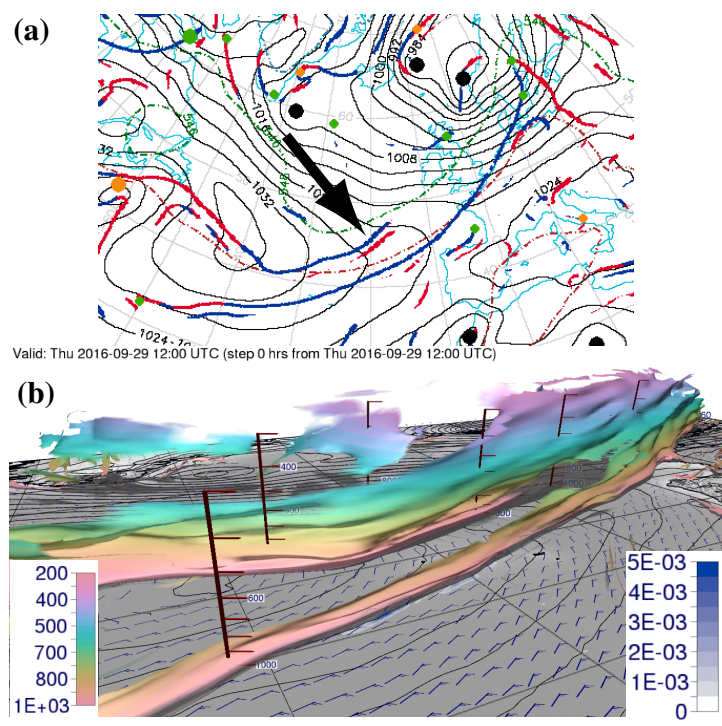

Fig. 12. Fronts of cyclone "Walpurga", at 12:00 UTC 29 September 2016. (a) ECMWF 2D objective surface fronts product. Note the seemingly detached front indicated by the arrow. (b) 3D fronts identified and visualized with our method, color denotes pressure elevation $(\mathrm{hPa})$. Note how the two fronts that are seemingly independent in the 2D image are actually connected at upper levels. netic or frontolytic, over-running and split), all of which can now be highlighted. Replies from research referred also to combining the 3D fronts with other variables and visualizations, such as front-normal winds, microphysical quantities, trajectories and vertical profiles. Interestingly, reactions to some plots varied markedly; one found Fig. 12 illuminating whilst another found it confusing. Evidently familiarization will be important.

\section{Discussion ANd Conclusion}

We have proposed a full 3D identification and visualization methodology for investigating atmospheric frontal structures, which are intrinsically 3D. Such analysis was not possible before, using classical 2D techniques. Our techniques include depiction of 3D frontal surfaces, depiction of frontal zones by means of normal curves, color-mapping of atmospheric quantities onto displayed features, and combination with further atmospheric fields and features. Frontal features of interest can be isolated interactively, and information about the distribution of atmospheric quantities within a frontal structure can be queried.

We briefly discuss our results. Our technique builds upon an established 2D method $[14,15]$ that has been run in operational environments for years, thereby achieving consistency with existing products. At the same time this might be considered a limitation, as somewhat different views exist within the meteorological community regarding how fronts should be defined (e.g., [42,49]). Nonetheless, our portrayed structures do make physical sense, they do conform, to a large degree, with conventional ideas, and they do provide, in addition, many new insights. Two real-world cases from the 2016 NAWDEX atmospheric field campaign have highlighted the value of our method for meteorological analysis and shown in one case (Walpurga) a structural feature not previously documented. With respect to lessons learned during the design stage, we note that in particular the use of transparency for "soft" filtering was much appreciated by the domain experts in the author team (after discovering the strong sensitivity of "hard" filtered fronts to thresholds). Also, the isolated depiction of individual fronts proved very beneficial to reduce cluttering and to permit focus.

In conclusion, we are confident that our method will facilitate many new and valuable studies in atmospheric research, and that it will also benefit operational forecasting, particularly for adverse weather which often relates to fronts. Newly identified frontal characteristics will be subject to further investigations in the context of NAWDEX and beyond. This will include evaluation of traditional ideas by comparison of conceptual models and observations to the multiple 3D structures identified by our approach. We are confident this will stimulate further meteorological research to help reconcile different views on fronts, and to improve representation of frontal processes in numerical models.

\section{ACKNOWLEDGMENTS}

The research leading to these results has been done within the subproject "Visualization of coherence and variation in meteorological dynamics" of the Transregional Collaborative Research Center SFB/TRR 165 "Waves to Weather" funded by the German Research Foundation (DFG). We are grateful to Sophia Schäfer for providing the "aquaplanet" dataset. ECMWF data has been kindly provided in the context of the ECMWF special project "Support Tool for HALO Missions". 


\section{REFERENCES}

[1] American Meteorological Society. "front". glossary of meteorology. http: //glossary.ametsoc.org/wiki/Front, 2018. Accessed 31 March 2018.

[2] M. J. Bader, G. S. Forbes, J. R. Grant, R. B. E. Lilley, and A. J. Waters. Images in weather forecasting : a practical guide for interpreting satellite and radar imagery. Cambridge University Press, 1995.

[3] G. Berry, M. J. Reeder, and C. Jakob. A global climatology of atmospheric fronts. Geophys. Res. Lett., 38(4):L04809+, Feb. 2011.

[4] J. Bjerknes and H. Solberg. Life cycles of cyclones and the polar front theory of atmospheric circulation. Geofys. Publ., 3:1-18, 1922.

[5] D. C. Breton. Note sur les lignes de faîte et de thalweg. Comptes rendus hebdomadaires des séances de l'académie des Sciences, 39:647-648, 1854.

[6] K. A. Browning and G. A. Monk. A simple model for the synoptic analysis of cold fronts. Quarterly Journal of the Royal Meteorological Society, 108(456):435-452, Apr. 1982.

[7] M. de Saint-Venant. Surfaces à plus grande pente constituées sur des lignes courbes. Bulletin de la Société Philomathématique de Paris, pp. 24-30, 1852 .

[8] D. Eberly. Ridges in Image and Data Analysis (Computational Imaging and Vision). Springer, 1996 ed., Sept. 1996.

[9] K. Engel, M. Hadwiger, J. Kniss, C. Rezk-Salama, and D. Weiskopf Real-Time Volume Graphics. A K Peters, Wellesley, Mass., July 2006.

[10] J. D. Furst and S. M. Pizer. Marching ridges. In 2001 IASTED International Conference on Signal and Image Processing, pp. 22-26, 2001.

[11] J. A. Grim, R. M. Rauber, M. K. Ramamurthy, B. F. Jewett, and M. Han. High-Resolution observations of the Trowal-Warm-frontal region of two continental winter cyclones. Monthly Weather Review, 135(5):1629-1646, May 2007.

[12] M. Han, S. A. Braun, Persson, and J.-W. Bao. Alongfront variability of precipitation associated with a midlatitude frontal zone: TRMM observations and MM5 simulation. Monthly Weather Review, 137(3):1008-1028, Mar. 2009.

[13] R. M. Haralick. Ridges and valleys on digital images. Computer Vision, Graphics, and Image Processing, 22(1):28 - 38, 1983.

[14] T. D. Hewson. Objective fronts. Met. Apps, 5(1):37-65, 1998.

[15] T. D. Hewson and H. A. Titley. Objective identification, typing and tracking of the complete life-cycles of cyclonic features at high spatial resolution. Met. Apps, 17(3):355-381, 2010.

[16] P. Hope, K. Keay, M. Pook, J. Catto, I. Simmonds, G. Mills, P. McIntosh, J. Risbey, and G. Berry. A comparison of automated methods of front recognition for climate studies: A case study in southwest western australia. Monthly Weather Review, 142(1):343-363, Jan. 2014.

[17] J. Jenkner, M. Sprenger, I. Schwenk, C. Schwierz, S. Dierer, and D. Leuenberger. Detection and climatology of fronts in a high-resolution model reanalysis over the alps. Meteorological Applications, 17(1):n/a, Mar 2009.

[18] M. Kašpar. Objective frontal analysis techniques applied to Extreme/Nonextreme precipitation events. 47(3):605-631, 2003

[19] M. Kern, T. Hewson, F. Sadlo, R. Westermann, and M. Rautenhaus. Robust detection and visualization of jet-stream core lines in atmospheric flow. IEEE Trans. Visual. Comput. Graphics, 24(1):893-902, 2018.

[20] G. Kindlmann. Semi-automatic generation of transfer functions for direct volume rendering. Master's thesis, Cornell University, USA, 1999.

[21] T. Lindeberg. Edge detection and ridge detection with automatic scale selection. 30(2):117-156, 1998.

[22] J. D. Locatelli, J. E. Martin, and P. V. Hobbs. A wide cold-frontal rainband and its relationship to frontal topography. Q.J.R. Meteorol. Soc., 120(516):259-275, Jan. 1994

[23] J. D. Locatelli, M. T. Stoelinga, M. F. Garvert, and P. V. Hobbs. The IMPROVE-1 storm of 1-2 february 2001. part i: Development of a Forward-Tilted cold front and a warm occlusion. Journal of the Atmospheric Sciences, 62(10):3431-3455, Oct. 2005.

[24] J. D. Locatelli, M. T. Stoelinga, and P. V. Hobbs. Re-examination of the split cold front in the british isles cyclone of 17 july 1980. Quarterly Journal of the Royal Meteorological Society, 131(612):3167-3181, Oct. 2005.

[25] W. E. Lorensen and H. E. Cline. Marching cubes: A high resolution 3D surface construction algorithm. In Proceedings of the 14th Annual Conference on Computer Graphics and Interactive Techniques, vol. 21 of SIGGRAPH '87, pp. 163-169. ACM, New York, NY, USA, July 1987.
[26] P. Majer. A statistical approach to feature detection and scale selection in images. Niedersächsische Staats-und Universitätsbibliothek, 2000

[27] J. E. Martin. Mid-Latitude Atmospheric Dynamics: A First Course. Wiley, 1 ed., June 2006.

[28] C. F. Mass. Synoptic frontal analysis: Time for a reassessment? Bulletin of the American Meteorological Society, 72(3):348-363, Mar. 1991.

[29] C. F. Mass and D. M. Schultz. The structure and evolution of a simulated midlatitude cyclone over land. Mon. Wea. Rev., 121(4):889-917, Apr. 1993.

[30] Met.3D contributors. Met.3D. http://met3d.wavestoweather.de, 2018. Accessed 31 March 2018.

[31] A. E. Milionis and T. D. Davies. The effect of the prevailing weather on the statistics of atmospheric temperature inversions. International Journal of Climatology, 28(10):1385-1397, Aug. 2008.

[32] K. C. Mulqueen and D. M. Schultz. Non-classic extratropical cyclones on met office sea-level pressure charts: double cold and warm fronts. Weather, 70(3):100-105, Mar. 2015.

[33] R. Peikert and F. Sadlo. Height ridge computation and filtering for visualization. In 2008 IEEE Pacific Visualization Symposium, pp. 119-126, March 2008.

[34] T. Pfaffelmoser, M. Reitinger, and R. Westermann. Visualizing the positional and geometrical variability of isosurfaces in uncertain scalar fields. Comput. Graphics Forum, 30(3):951-960, June 2011.

[35] J. G. Pinto, I. n. Gómara, G. Masato, H. F. Dacre, T. Woollings, and R. Caballero. Large-scale dynamics associated with clustering of extratropical cyclones affecting western europe. Journal of Geophysical Research: Atmospheres, 119(24):13,704-13,719, Dec. 2014.

[36] M. Rautenhaus, M. Bottinger, S. Siemen, R. Hoffman, R. M. Kirby, M. Mirzargar, N. Rober, and R. Westermann. Visualization in meteorology - a survey of techniques and tools for data analysis tasks. IEEE Trans. Visual. Comput. Graphics, preprint:1, 2018.

[37] M. Rautenhaus, C. M. Grams, A. Schäfler, and R. Westermann. Threedimensional visualization of ensemble weather forecasts - Part 2: Forecasting warm conveyor belt situations for aircraft-based field campaigns. Geosci. Model Dev., 8(7):2355-2377, 2015.

[38] M. Rautenhaus, M. Kern, A. Schäfler, and R. Westermann. Threedimensional visualization of ensemble weather forecasts - Part 1: The visualization tool Met.3D (version 1.0). Geosci. Model Dev., 8(7):2329_ 2353, 2015.

[39] R. J. Renard and L. C. Clarke. Experiments in numerical objective frontal analysis 1. Monthly Weather Review, 93(9):547-556, Sept. 1965.

[40] F. Sadlo and R. Peikert. Efficient visualization of Lagrangian coherent structures by filtered AMR ridge extraction. IEEE Transactions on Visualization and Computer Graphics, 13(6):1456-1463, Nov 2007.

[41] J. Sahner, T. Weinkauf, N. Teuber, and H. C. Hege. Vortex and strain skeletons in Eulerian and Lagrangian frames. IEEE Transactions on Visualization and Computer Graphics, 13(5):980-990, Sept 2007.

[42] F. Sanders. A proposed method of surface map analysis. Monthly Weather Review, 127(6):945-955, June 1999.

[43] F. Sanders and C. A. Doswell. A case for detailed surface analysis. Bulletin of the American Meteorological Society, 76(4):505-521, Apr. 1995.

[44] S. A. K. Schäfer and A. Voigt. Radiation weakens idealized midlatitude cyclones. Geophysical Research Letters, 45(6):2833-2841, Mar. 2018.

[45] A. Schäfler, G. Craig, H. Wernli, P. Arbogast, J. D. Doyle, R. McTaggartCowan, J. Methven, G. Rivière, F. Ament, M. Boettcher, M. Bramberger, Q. Cazenave, R. Cotton, S. Crewell, J. Delanoë, A. Dörnbrack, A. Ehrlich, F. Ewald, A. Fix, C. M. Grams, S. L. Gray, H. Grob, S. Groß, M. Hagen, B. Harvey, L. Hirsch, M. Jacob, T. Kölling, H. Konow, C. Lemmerz, O. Lux, L. Magnusson, B. Mayer, M. Mech, R. Moore, J. Pelon, J. Quinting, S. Rahm, M. Rapp, M. Rautenhaus, O. Reitebuch, C. A. Reynolds, H. Sodemann, T. Spengler, G. Vaughan, M. Wendisch, M. Wirth, B. Witschas, K. Wolf, and T. Zinner. The north atlantic waveguide and downstream impact experiment. Bull. American Meteor. Soc., preprint, 2018

[46] S. Schemm, L. Nisi, A. Martinov, D. Leuenberger, and O. Martius. On the link between cold fronts and hail in switzerland. Atmos. Sci. Lett. 17(5):315-325, May 2016.

[47] S. Schemm and M. Sprenger. Frontal-wave cyclogenesis in the north atlantic -a climatological characterisation. Q.J.R. Meteorol. Soc., 141(693):2989-3005, Oct. 2015

[48] D. M. Schultz and C. F. Mass. The occlusion process in a midlatitude cyclone over land. Mon. Wea. Rev., 121(4):918-940, Apr. 1993.

[49] D. M. Schultz and G. Vaughan. Occluded fronts and the occlusion pro- 
cess: A fresh look at conventional wisdom. Bulletin of the American Meteorological Society, 92(4):443-466, Apr. 2011.

[50] D. M. D. Schultz and W. Blumen. SYNOPTIC METEOROLOGY - Fronts, pp. 337-343. Elsevier, 2015.

[51] S. C. Shadden, F. Lekien, and J. E. Marsden. Definition and properties of Lagrangian coherent structures from finite-time Lyapunov exponents in two-dimensional aperiodic flows. Physica D: Nonlinear Phenomena, 212(3-4):271 - 304, 2005.

[52] M. A. Shapiro and D. Keyser. Fronts, jet streams, and the tropopause. In C. Newton and E. O. Holopainen, eds., Extratropical Cyclones. The Erik Palmen Memorial Volume, pp. 167-191. American Meteorological Society, Boston, MA, 1990.

[53] I. Simmonds, K. Keay, and J. A. Tristram Bye. Identification and climatology of southern hemisphere mobile fronts in a modern reanalysis. $J$. Climate, 25(6):1945-1962, Sept. 2011.

[54] R. Stauffer, G. J. Mayr, M. Dabernig, and A. Zeileis. Somewhere over the rainbow: How to make effective use of colors in meteorological visualizations. Bull. Amer. Meteor. Soc., 96(2):203-216, Feb. 2015.

[55] R. C. Strawn, D. N. Kenwright, and J. Ahmad. Computer visualization of vortex wake systems. AIAA journal, 37(4):511-512, 1999.

[56] R. Stull. Practical Meteorology: An Algebra-based Survey of Atmospheric Science. Creative Commons License, Copyright 2017, 2018 by Roland Stull, University of British Columbia, Vancouver, BC, v1.02b ed., 2017. 